\title{
artigo
}

Pugliesi, C.H.H.; Fabene, M.R.; Marques, F.R.D.M.; Mareze, M.; Salci, M.A.; Charlo, P.B.;

Estudo epidemiológico de gestantes atendidas pelo sistema único de saúde com ênfase na toxoplasmose

\section{Estudo epidemiológico de gestantes atendidas pelo sistema único de saúde com ênfase na toxoplasmose}

\author{
Epidemiological study of pregnant women served by the public health system with emphasis on toxoplasmosis \\ Estudio epidemiológico de mujeres embarazadas servidas por el sistema de salud único con énfasis en la toxoplasmosis
}

\begin{abstract}
RESUMO
Objetivo: identificar a correlação dos fatores de risco associados à incidência da toxoplasmose nas gestantes atendidas pelo Sistema Único de Saúde em município de médio porte no Estado do Paraná. Método: estudo transversal quantitativo, de janeiro a fevereiro de 2019, com gestantes acompanhadas em 17 UBS, as quais responderam questionário socioeconômico, hábitos alimentares, de higiene e aspectos relacionados ao ambiente e à doença. Análise estatística pelo programa Epilnfo versão 7.0. Incluídas no estudo 143 gestantes. Resultados: a análise mostrou que o consumo de verduras/legumes crus ( $p=0,9 ; 0 R=1,71$ ), carne crua ou mal passada $(p=0,87 ; O R=0,82)$, possuir gato na residência $(p=0,56 ; O R=1,16)$, ter baixa escolaridade $(p=0,17$; $\mathrm{OR}=0,36)$ e ter baixa renda familiar $(\mathrm{p}=0,45 ; \mathrm{OR}=0,51)$ não teve associação significativa com a sororeatividade para Toxoplasma gondii. Possuir cachorro na residência $(p<0,001 ; 0 R=0,16)$ mostrou uma relação positiva entre dados, porém sem representatividade de risco para essas gestantes. Conclusões: necessidade de estudos que auxiliem na formulação de novas estratégias para o controle e tratamento desta infecção.
\end{abstract}

DESCRITORES: Complicações Infecciosas na Gravidez; Cuidado pré-natal; Saúde pública.

\section{ABSTRACT}

Objective: to identify the correlation of risk factors associated with the incidence of toxoplasmosis in pregnant women attended by the Public Health System in a medium-sized city, in the State of Parana. Method: cross-sectional quantitative study from January to February 2019, with pregnant women followed up in $17 \mathrm{BHU}$, which answered questionnaire socio-economic, food, hygiene habits and aspects related to environment and the disease. Statistical analysis by using the Epilnfo version 7.0 program. 143 pregnant women were included in the study. Results: the analysis showed that the consumption of raw vegetables ( $p=0.9$; $O R=1.71)$ and raw or undercooked meat $(p=0.87 ; O R=0.82)$, having a cat at home $(p=0.56 ; O R=1.16)$, having low level of education ( $p=0.17 ; O R=0.36)$ and having a low family income $(p=0.45 ; O R=0.51$ ) did not have significant association with Toxoplasma gondii seroreactivity. Owning a dog at home $(\mathrm{p}<0.001 ; \mathrm{OR}=0.16)$ presented a positive relationship between data, but with no representativeness of risk for these pregnant women. Conclusions: the need for further studies which may assist the formulation of new strategies for control and treatment of this infection.

DESCRIPTORS: Pregnancy Complications, Infectious; Prenatal Care; Public Health.

\section{RESUMEN}

Objectivo: identificar la correlación de factores de riesgo asociados a la incidencia de toxoplasmosis en gestantes atendidas por el Sistema Único de Salud en un municipio mediano del Estado de Paraná. Método: estudio cuantitativo, transversal, de enero a febrero de 2019, con gestantes seguidas en 17 UBS, cuáles respondidas cuestionario socioeconómico, hábitos alimentarios, higiene y aspectos relacionados con el medio ambiente y la enfermedad. Análisis estadístico mediante el programa Epilnfo versión 7.0. Se incluyeron 143 mujeres embarazadas en el estudio. Resultados: el análisis mostró que el consumo de verduras crudas $(p=0,9 ; O R=1,71)$ y carne cruda o poco cocida $(p=0,87 ; O R=0,82)$, tener un gato en casa $(p=0,56 ; O R=1,16)$, tener bajo nivel educativo $(p=0,17 ; O R=0,36)$ y tener bajos ingresos familiares $(p=0,45 ; O R=0,51)$ no tenían asociación significativa con serorreactividad para Toxoplasma gondii. Tener un perro en casa $(p<0,001 ; O R=0,16)$ mostró una relación positiva entre los datos, pero sin representatividad de riesgo para estas gestantes. Conclusiones: la necesidad de estudios con el fin de ayudar en la formulación de nuevas estrategias para el control y tratamiento de esta infección.

DESCRIPTORES: Complicaciones infecciosas en el embarazo; Cuidado prenatal; Salud pública.

RECEBIDO EM: 04/09/2020 APROVADO EM: 29/09/2020 
Caio Henrique Hashimoto Pugliesi

Acadêmico do curso de medicina da Unicesumar. ORCID: 0000-0002-7467-0862

\section{Márcio Renan Fabene}

Acadêmico do curso de medicina da Unicesumar.

ORCID: 0000-0002-6072-1964

\section{Francielle Renata Danielli Martins Marques}

Mestranda em Enfermagem pelo Programa de Pós Graduação em Enfermagem pela Universidade Estadual de Maringá. ORCID: 0000-0002-8578-9615

\section{Marcelle Mareze}

Professora do curso de Enfermagem na Faculdade de Tecnologia do Vale do Ivaí. ORCID: 0000-0001-7266-5851

\section{Maria Aparecida Salci}

Doutora em Enfermagem, Docente do Programa de Pós Graduação em Enfermagem pela Universidade Estadual de Maringá. ORCID: 0000-0002-6386-1962

\section{Patrícia Bossolani Charlo}

Professora do curso de Medicina da Unicesumar.

ORCID: 0000-0002-8262-2086

\section{INTRODUÇÃO}

A toxoplasmose resulta da ingesta de oocistos em carne crua ou semicozida, contato com fezes de gatos infectadas com o oócito e transmissão vertical $^{1}$. Na gestação, a principal forma de contaminação ocorre por meio do consumo de carne crua ou mal cozida, água contaminada, ou pela má higienização das mãos após contato com fezes de felinos em solo, areia ou lixo' ${ }^{2}$.

A alta incidência de transmissão congênita, que varia entre quatro a 10 casos para cada 10 mil nascidos vivos no Brasil, pode causar graves complicações, incluindo alterações oculares (coriorretinite), neurológicas (encefalite, microcefalia e macrocefalia), sistêmicas (hepatomegalia, icterícia) $)^{3-4}$, fetal (aborto ou malformações) ou ainda sequelas graves tardias, mesmo em crianças assintomáticas ao nascimento ${ }^{5-6}$.

O Brasil está entre os países que apresentam as maiores taxas de incidências de toxoplasmose no mundo, o que resultou em reestruturação na vigilância epidemiológica por meio de mudanças na notificação compulsória de toxoplasmose gestacional e congênita, e padronização da investigação dos casos suspeitos e confirmados ${ }^{7}$.
No cenário nacional, cerca de 60 a 75\% das mulheres em idade fértil apresentam imunidade para toxoplasmose (IgG reagente e $\operatorname{IgM}$ não reagente) ${ }^{8}$. A susceptibilidade ocorre quando o resultado sorológico indica $\operatorname{IgG}$ e IgM não reagentes; e uma possível infecção ativa, quando a sorologia apresenta $\operatorname{IgG}$ reagente ou não reagente e IgM reagente ${ }^{8}$.

Em um município do norte do Paraná, verificou-se que mais de $50 \%$ das gestantes são susceptíveis à toxoplasmose, elevando a incidência de casos congênitos 5 . Ressalta-se que o conhecimento do cenário epidemiológico torna-se essencial para traçar medidas de controle de prevenção desta doença ${ }^{1}$.

A triagem materna de doenças infecciosas durante o pré-natal possibilita o diagnóstico e o tratamento precoce, tendo em vista que a transmissão de infecções verticais é responsável pelo aumento da morbimortalidade do binômio mãe-filho, tornando-se um grave problema de Saúde Pública no Brasil ${ }^{9}$.

Nesse sentido, o governo federal implementou a Rede Cegonha a partir da Portaria ${ }^{\circ} 1.459$, de 24 de junho de $2011^{10}$ como forma de complementar o Programa de Humanização no Pré-natal e Nascimento $(\mathrm{PHPN})^{10,11}$. E o Estado do Paraná viu a ne- cessidade de criar, em 2013, a Rede Mãe Paranaense (RMP) abrangendo um conjunto de ações envolvendo a captação precoce da gestante, acompanhamento no pré-natal, realização de consultas e exames, estratificação de risco com vinculação à maternidade para o parto, sendo que a toxoplasmose identificada no pré-natal é critério de estratificação de risco, e o acompanhamento considerado de alto risco ${ }^{12}$.

Dada a importância de que seja realizado o diagnóstico precoce da Toxoplasmose, a Linha Guia da RMP recomenda a solicitação da sorologia (IgG e $\operatorname{IgM}$ ) no primeiro atendimento, sendo repetido por trimestre se a gestante for suscetível ${ }^{12}$. A confirmação da suscetibilidade ou do diagnóstico de infecção aguda contribui para a diminuição da primo-infecção, da transmissão vertical e das sequelas, principalmente ao feto, já que permite a prevenção primária e o tratamento precoce ${ }^{13}$.

O estabelecimento de protocolos permite a coleta de informação epidemiológica para identificar a fonte de infecção, modo de transmissão, grupos vulneráveis à doença, fatores de risco para o adoecimento; e, confirmar o diagnóstico e determinar as principais características epidemiológicas, resultando em medidas 
de prevenção, controle e tratamento para impedir a ocorrência de novos casos ${ }^{14}$.

Diante desse contexto e considerando a relevância em conhecer os fatores associados à infecção por toxoplasmose em uma população específica, com a finalidade de produzir dados que possam auxiliar a vigilância epidemiológica na construção de medidas preventivas e curativas, emergiu a seguinte questão de pesquisa: a adesão de comportamentos de risco está associado à presença de $\operatorname{IgM}$ positiva para toxoplasmose durante à gestação?

Para tanto, o objetivo deste estudo foi identificar a correlação dos fatores de risco associados à incidência da toxoplasmose nas gestantes atendidas pelo Sistema Único de Saúde (SUS) em um município de médio porte localizado no Estado do Paraná.

\section{MÉTOdO}

Trata-se de um estudo transversal, de caráter quantitativo, realizado no período de janeiro a fevereiro de 2019, em 17 Unidades Básicas de Saúde (UBS) situadas em um município do Estado do Paraná.

As 17 UBS foram escolhidas aleatoriamente visando uma amostra homogênea da população. Nestas UBS, foram selecionadas 143 gestantes que realizaram o pré-na-

\section{Tabela1. Caracterização dos sujeitos da pesquisa. Maringá, 2019.}

\section{Variáveis sociais}

Estado civil

Casada
54

31

Solteira

união estável

Divorciada

Raça

Branca

Parda

Preta

Amarela

Indígena

Faixa etária

$\leq 18$ anos

$\geq 19$ e $\leq 59$

Escolaridade

$\leq 9$ anos

$\geq 10$ e $\leq 13$ anos

$\geq 14$ anos

Renda familiar

$<1$ salário mínimo

$\geq 1$ e $\leq 3$ salários mínimos

$>3$ salários mínimos

Responsável pela renda

Gestante

Esposo

Familia

47

5

65

53

\section{Frequência $(n=143)$}

$\%$

39,4

22,6

34,3

3,6

47,4

*Salário mínimo vigente no ano de 2019: R\$998,00 tal integralmente no SUS. Como critério de inclusão: ter realizado, no mínimo, 7 consultas de pré-natal no serviço de saúde.

A coleta de dados ocorreu por meio de entrevistas a partir de um questionário estruturado com questões que versavam sobre: grau de instrução, renda familiar, moradia/saneamento básico, investigação sobre contato com gatos, hábitos de higiene dos alimentos e conhecimento sobre prevenção da toxoplasmose.

Os dados foram organizados por meio do programa Microsoft Excel, 2010, posteriormente analisados utilizando estatística descritiva, expressa sob a forma de frequência absoluta e relativa. A medida da força de associação entre as variáveis foi obtida pelo programa EpiInfo versão 7.0 CDC-Atlanta pela regressão logística não condicional, que identificou inicialmente, por meio da razão de chances (Odds Ratio - OR), o valor da OR bruta, para as variáveis de cada bloco que apresentaram associação estatística significativa $(p<0,05)$. O controle dos possíveis fatores de confusão existentes nas associações obtidas na análise univariada foi conseguido por meio da técnica de análise multivariada, em que foi obtido o valor da OR ajustada.

$\mathrm{O}$ presente estudo obedeceu às recomendações das Resoluções 466/2012 e 510/2016 15 do Conselho Nacional de Saúde que regulamentam a pesquisa envolvendo seres humanos e todas as gestantes participantes assinaram Termo de Consentimento Livre e Esclarecido (TCLE). Foi submetido e aprovado pelo do Comitê de Ética em Pesquisa da Unicesumar segundo parecer número 3.031.898, CAAE número 02582618.3.0000.5539.

\section{RESULTADOS}

Foram incluídas no estudo 143 gestantes. A maioria era casada (54-39,4\%), branca $(65-47,4 \%)$, com faixa etária acima de 19 anos (137-95,8\%), com escolaridade que variou entre 10 a 13 anos de estudo (79-55,2\%), com renda familiar entre um a três salários mínimos (80-63,4\%), sendo o esposo (101-73,7\%) o principal responsável pela renda familiar, conforme Tabela 1. 
Tabela2. Caracterização dos hábitos de higiene. Maringá, 2019.

$$
\text { Hábitos de higiene }
$$

Frequência $(n=143)$

$\%$

Lavar a tábua após cortar carne

$\begin{array}{lcc}\text { Com água } & 9 & 6,2 \\ \text { Água e sabão } & 132 & 92,3 \\ \text { Não lava } & 2 & 1,4 \\ \text { Comer verduras e legumes crus } & 60 & 41,9 \\ \text { Todos os dias } & 62 & 43,3 \\ \text { Duas vezes na semana } & 21 & 14,6 \\ \text { Não come } & 24 & 16,9 \\ \text { Comer carne crua ou mal passada } & 118 & 83,1 \\ \text { Sim } & & 11,8 \\ \text { Não } & 17 & 88,1 \\ \text { Quibe cru } & 126 & 27,2 \\ \text { Sim } & & 72,7 \\ \text { Não } & 39 & \\ \text { Churrasco mal passado } & 104 & \\ \text { Sim } & & \\ \text { Não } & & \end{array}$

Tabela 3. Caracterização dos aspectos relacionados à doença. Maringá, 2019.

Aspectos relacionados à doença

Conhecimento prévio sobre Toxoplasmose

$\operatorname{Sim}$
113

30

Não

Realizado sorologia para Toxoplasmose

Sim

91

Não

Não sabe

21

Resultado IgM para Toxoplasmose

Positivo

13

Negativo

72

Não sabe

54

Fez tratamento para Toxoplasmose

Sim

2

132

Não

\section{Tabela 4. Caracterização dos aspectos ambientais. Maringá, 2019.}

$$
\text { Aspectos ambientais }
$$

Frequência $(n=143)$

$\%$

\section{Gato na residência}

Sim

Não
79,1

63,6

21,6

14,6

9,3

51,8

38,8

$\%$

20,9

\section{3}

8,8

1,5

98,5
Em relação aos hábitos de higiene, a maioria afirmou lavar a tábua de carne com água e sabão (132-92,3\%), comer verduras ou legumes crus duas vezes na semana (62-43,3\%), não comer carne crua ou mal passada $(118-83,1 \%)$, não comer quibe cru $(126-88,1 \%)$ e não comer churrasco mal passado (104$72,7 \%)$, como descritos na Tabela 2.

Quanto à doença, a maioria afirmou ter conhecimento prévio sobre a Toxoplasmose $(113-79,1 \%)$, ter realizado sorologia (91-63,6\%), sendo que pouco mais da metade $(72-51,8 \%)$ tinha o diagnóstico negativo no momento e relataram não ter realizado tratamento específico para a doença nessa gestação (132-98,5\%), conforme Tabela 3.

Quanto às questões ambientais, de convivência com animais domésticos, a maioria possuía cachorro (88-62,4\%), seguida da presença de gato na residência (21-14,7\%), conforme Tabela 4.

A análise mostrou que o consumo de verduras e legumes crus $(p=0,9$; $\mathrm{OR}=1,71)$, carne crua ou mal passada ( $\mathrm{p}=0,87 ; \mathrm{OR}=0,82)$, possuir gato na residência $(p=0,56 ; O R=1,16)$, ter baixa escolaridade $(p=0,17 ; O R=0,36)$ e ter baixa renda familiar $(\mathrm{p}=0,45 ; \mathrm{OR}=0,51)$ não teve associação significativa com a sororeatividade para Toxoplasma gondii nessas gestantes. Quanto à variável possuir cachorro na residência $(p<0,001$; $\mathrm{OR}=0,16$ ), houve uma relação positiva, porém sem representatividade de risco para essas gestantes (Tabela 5).

\section{DISCUSSÃO}

A caracterização sociodemográfica, hábitos de higiene e aspectos ambientais das gestantes de alto risco são dados que podem auxiliar na construção de um plano epidemiológico para prevenção de agravos, mas que seja condizente com a realidade apresentada ${ }^{16}$. Neste estudo, predominaram gestantes casadas ou com união estável, evidenciando que o planejamento de uma gestação tem ocorrido dentro de um relacionamento mais consolidado ${ }^{17-18}$. A presença da raça branca 


\section{Cachorro na residência}

Sim

88

62,4

Não

53

37,6

Tabela 5. Associação entre fatores de risco e sororeatividade para T. gondii. Maringá, 2019.

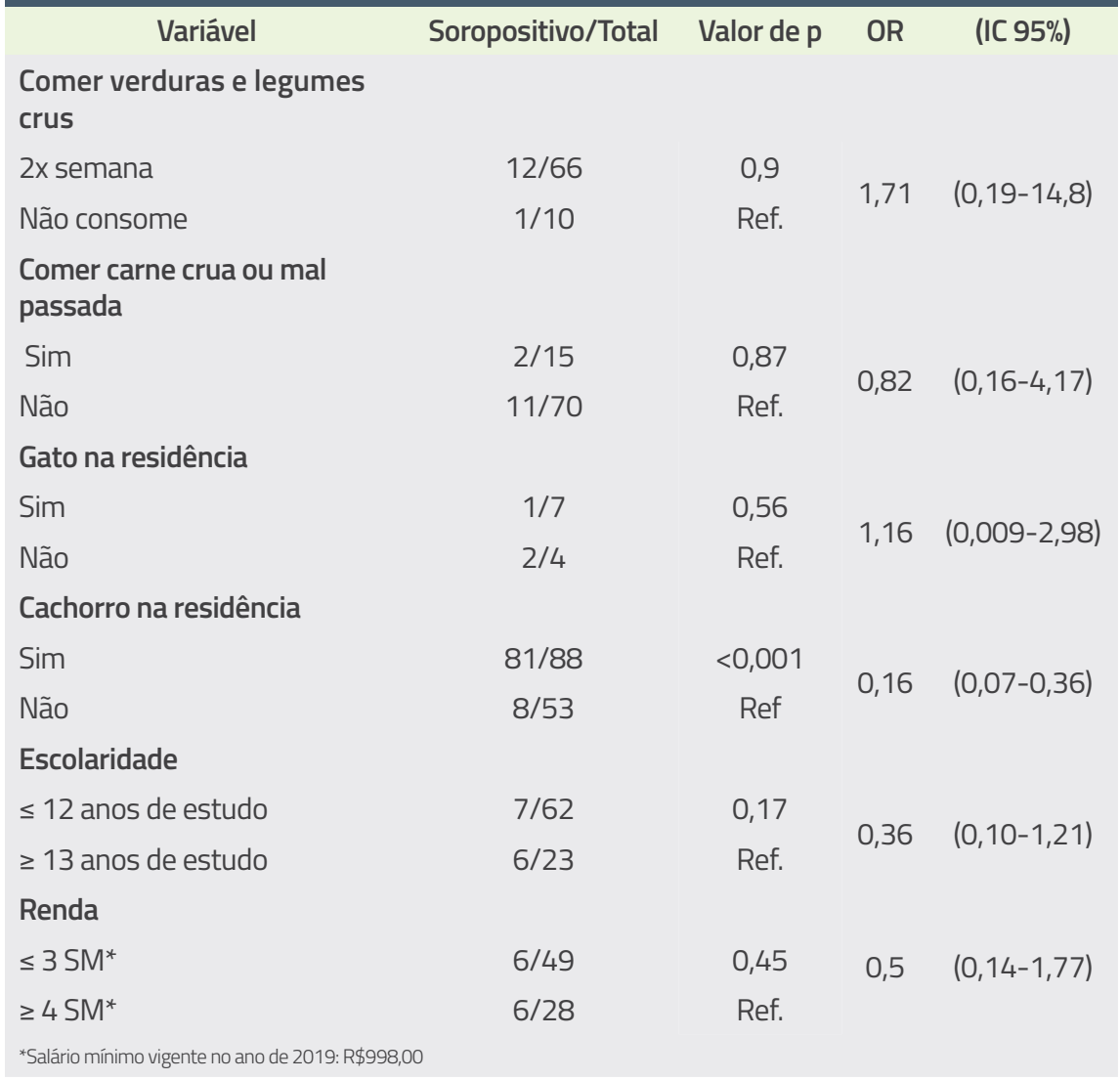

corrobora com estudo ${ }^{18}$ sobre pré-natal de alto risco realizado em região próxima ao presente estudo, evidenciando ser uma característica étnica da população deste região.

Com relação à faixa etária das gestantes, existe uma relação entre a soroprevalência e o acréscimo da idade ${ }^{2,19}$. Pessoas com mais de 18 anos, como encontrado neste estudo, possuem maior risco de serem soropositivas para IgG por terem maior tempo de exposição ao T. gondii no ambiente ${ }^{20}$.

O baixo nível educacional e econômico $18,{ }^{20-23}$, como evidenciado nesta pesquisa, também são fatores de risco associados com a infecção por T. gondii, demonstrando que as gestantes com esses fatores de risco devem ser monitoradas mais cuidadosamente durante as consultas de pré-natal. Gestantes com maiores níveis educacionais demonstram terem melhores hábitos de higiene e melhor acesso às informações ${ }^{24-26}$.

Em relação aos hábitos de vida e higiene, a grande maioria das gestantes referiu lavar a tábua de carnes com água e sabão. O hábito de lavar os utensílios de cozinha com água e sabão esteve associado estatisticamente como fator de proteção à doença $a^{23}$.

Este estudo encontrou um baixo consumo de legumes e verduras crus, sendo um dado considerado satisfatório já que o consumo desses alimentos teve associação significativa com a so- roreatividade para $T$. gondii em gestantes em estudo semelhante ${ }^{24}$. Em relação ao consumo de carne crua, quibe cru e churrasco mal passado, tais hábitos não foram significativamente associados à infecção pelo patógeno em questão, assim como encontrados em outros estudos brasileiros ${ }^{20,25}$. Em revisão sistemática $^{27}$ realizada sobre a prevalência de $T$. gondii em carnes destacou que a carne de gado, geralmente são as que menos contêm oocistos, com prevalência inferior às de porco e ovelha.

O pré-natal é o momento ideal para orientação de medidas preventivas das doenças de transmissão materno-fetal, como a toxoplasmose, com medidas que vão desde a orientação até a realização da sorologia ${ }^{21}$, sendo referido pelas gestantes da amostra estudada conhecimento prévio sobre a doença e a importância da realização de sorologia para toxoplasmose, o que difere dos dados encontrados em estudo sobre o conhecimento das gestantes no Maranhão ${ }^{23}$.

Em revisão de literatura sobre a qualidade do pré-natal no Brasil ${ }^{28}$, destacam-se como fatores que contribuem para a baixa qualidade da assistência a não realização dos exames laboratoriais de rotina e a falta de orientações durante as consultas. $\mathrm{O}$ conhecimento sobre a gestação de alto risco, bem como as doenças pré-existentes, são informações transmitidas durante uma consulta de pré-natal de qualidade, no qual o enfermeiro busca realizar a escuta qualificada, identificar os problemas e traçar um plano de cuidados individualizado para a gestante atendida. Nesse estudo, a prevalência de anticorpos IgM em mulheres grávidas foi de 9,3\%, superiores a outros estudos realizados no Brasil ${ }^{21,24}$. Ressalta-se a importância do trabalho preventivo e de promoção à saúde, com investimentos em programas educacionais como estratégia em Saúde Pública para prevenção de toxoplasmose ${ }^{24}$.

Com relação à convivência com animal de estimação, a maioria das gestantes o possuía, sendo predominantemente a criação do cão. A toxoplasmose é 
uma zoonose, portanto, transmitida ao homem pelos animais sendo que os cães podem atuar como vetores mecânicos de formas infectantes do $T$. gondii aderidos aos pêlos ${ }^{24}$.

Em relação às associações entre os fatores de risco e a sororeatividade para T. gondii, destaca-se que não há evidências entre o patógeno e os diversos hábitos de comportamento, como consumir verduras e legumes crus, consumir carne crua ou mal passada, possuir gato, corroborando com achados em estudos anteriores ${ }^{2,20,24-25}$.

Houve uma relação positiva sem representatividade de risco para essas gestantes, porém é sabido que a soroprevalência do $\mathrm{T}$. gondii pode variar de $5 \%$ a $84 \%$ dependendo da região geográfica e idade do cão ${ }^{29}$, sendo maior o risco de exposição para cães vadios do que para cães domésticos devido ao seu estilo de vida livre, que permite contato com outros hospedeiros intermediários e definitivos ${ }^{30}$.

As características sociodemográficas são apontadas por evidenciar fatores de risco para toxoplasmose, sendo citadas, principalmente a baixa renda 22 e a escolaridade ${ }^{21}$. Porém, nessa análise não se evidenciou associação significativa em relação a esses aspectos como nos estudos anteriores.

Cabe ressaltar que apesar da não associação estatística ao perfil sociodemográfico, relatos anteriores demonstraram que a escolaridade é um fator de risco importante para a ocorrência da toxoplasmose, e em geral, as pessoas com maior nível de escolaridade têm mais conhecimento sobre a infecção e os métodos de prevenção ${ }^{18,20-23}$.

Tendo em vista a alta incidência de Toxoplasmose gestacional no Brasil e, consequentemente, as mudanças nas políticas públicas no pré-natal para investigação e tratamento precoce desta infecção, estudos que visam identificar e correlacionar os fatores de risco às gestantes infectadas pelo t. gondii contribuem para fortalecer as ações de vigilância epidemiológica e cuidados no pré-natal subsidiando a formulação de protocolos assistenciais.

\section{CONCLUSÃO}

Foi possível concluir que as gestantes praticavam adequadamente os comportamentos preventivos, como lavar a tábua de carne com água e sabão, evitavam carne crua ou mal passada, assim como os alimentos que a continham, possuíam conhecimento prévio sobre a toxoplasmose, haviam realizado sorologia para a doença e não possuíam gato na residência.

As características sociodemográficas e de alguns hábitos de higiene não apresentaram associação significativa para o risco de toxoplasmose entre as gestantes, porém isto não exclui a possibilidade desses componentes propiciarem o contato com o T. gondii durante a gestação.

Reforça-se a necessidade de mais estudos sobre essa doença, em especial, associando os fatores de risco, para que possam auxiliar a formulação de novas estratégias para o controle e tratamento desta infecção.

\section{AGRADECIMENTOS}

Aos colaboradores e ao apoio financeiro através de bolsa de iniciação científica concedida pelo PIBIC/UniCesumar ao discente Caio Henrique Hashimoto Pugliesi durante a realização deste trabalho.

\section{REFERÊNCIAS}

1. Oliveira GMS de, Simões JM, Schaer RE, Freire SM, Nascimento RJM, Pinheiro AMC de M, Carvalho SMS, Mariano APM, Carvalho $\mathrm{RC}$ de, Munhoz AD. Frequência e fatores associados à infecção por Toxoplasma gondii em gestantes e seus animais de estimação em Ilhéus, Bahia, Brasil. Rev Soc Bras Med Trop 2019; 52.: e20190250.

2. Sharifi K, Hosseini Farash BR, Tara F, Khaledi A, Sharifi K, Shamsian S. Diagnosis of Acute Toxoplasmosis by IgG and IgM Antibodies and IgG Avidity in Pregnant Women from Mashhad, Eastern Iran. Iran J Parasitol. 2019; 14 (4): 639-645.

3. Brasil. Ministério da Saúde. Secretaria de Atenção à Saúde. Departamento de Ações Programáticas Estratégicas. Gestação de alto risco: manual técnico / Ministério da Saúde, Secretaria de Atenção à Saúde, Departamento de Ações Programáticas Estratégicas. - 5. ed. - Brasília: Editora do Ministério da Saúde, 2012. Acesso em: 27 jul 2019. Disponível em:<http:// bvsms.saude.gov.br/bvs/publicacoes/manual_tecnico_gestacao_alto_risco.pdf.
4. Universidade Federal do Rio Grande do Sul. Grupo de Trabalho Toxoplasmose Gestacional e Congênita SES/RS. Telecondutas: toxoplasmose na gestação. Universidade Federal do Rio Grande do Sul, 2019.

5. Capobiango JD, Breganó RM, Mori FMRL, Navarro IT, Campos JSA, Tatakihara LT Talizin TB, Santos M dos, Pereira TRG, Narciso SG, Reiche EMV. Gestational and congenital toxoplasmosis: a practical approach to disease notification. Epidemiol. Serv. Saúde [Internet]. 2016 Mar [acesso em 25 jul 2019]; 25( 1): 187-194. Disponivel em: http://www.scielo.br/scielo.php?script=sci_arttext\&pid=S2237-96222016000100187\&lng=en. http://dx.doi. org/10.5123/s1679-49742016000100020

6. Wallon M, Peyron F. Congenital Toxoplasmosis: A Plea for a Neglected Disease. Pathogens 2018, 7, 25.

7. Brasil. Ministério da Saúde. Secretaria de Vigilância em Saúde. Departamento de Vigilância das Doenças Transmissíveis. Protocolo de Notificação e Investigação: Toxoplasmose gestacional e congênita [recurso eletrônico]/ Ministério da Saúde, Secre- 


\section{REFERÊNCIAS}

taria de Vigilância em Saúde, Departamento de Vigilância das Doenças Transmissíveis. - Brasília : Ministério da Saúde, 2018.

8. Furini AA da C, Gadotti F da C, Bertasso-Borges MS, Pignata RCM, Sanches RMS, Faitorone NC, Santos I dos, Maschio-Lima TA, Cavasini CE, Machado RLD. Soroprevalência de anticorpos anti-toxoplasma gondii em amostras de gestantes no pré-natal. RBCS [Internet]. $16^{\circ}$ de março de 2016 [acesso em 25 mai 2020];19(3):199-04. Disponível em: https://periodicos.ufpb.br/ ojs2/index.php/rbcs/article/view/20576

9. Pedro SAPS, Silva CAL da, Rebouças MC, Costa MFD. Triagem de infecções pré-natais nas macrorregiões sul e sudoeste do estado da Bahia, Brasil: detectada em filtro de papel. Revista Brasileira de Saúde Materno Infantil, 2019; 19(3):681-690.

10. Brasil. Portaria n. 1.459/GM/MS, de 24 de junho de 2011: Institui, no âmbito do Sistema Único de Saúde - SUS - a Rede Cegonha. Brasília: Ministério da Saúde; 2011.

11. Martinelli KG, Neto ETS, Gama SGN da, Oliveira AE. Adequação do processo da assistência pré-natal segundo os critérios do Programa de Humanização do Pré-natal e Nascimento e Rede Cegonha. Rev. Bras. Ginecol. Obstet. 2014; 36(2): 56-64.

12. Paraná. Secretaria de Estado da Saúde. SESA. Linha Guia Rede Mãe Paranaense. 7a ed. 2018. Acesso em: 23 jul 2019. Disponivel em: <http://www.saude.pr.gov.br/arquivos/File/LinhaGuiaMaeParanaense_2018.pdf>..

13. Brasil. Ministério da Saúde. Secretaria de Atenção à Saúde. Departamento de Atenção Básica. Atenção ao pré-natal de baixo risco / Ministério da Saúde. Secretaria de Atenção à Saúde. Departamento de Atenção Básica. - 1. ed. rev. - Brasília: Editora do Ministério da Saúde, 2013. Acesso em 02 mar 2020. Disponível em: < https://central3.to.gov.br/arquivo/404128/>.

14. Brasil. Ministério da Saúde. Investigação epidemiológica de Casos, Surtos e Epidemias In: Guia de vigilância em saúde. 1. ed. atual. Brasília: Ministério da Saúde, 2017.

15. Brasil. Ministério da Saúde. Conselho Nacional de Saúde. Resolução N. 466, de 12 de dezembro de 2012 - Diretrizes e normas regulamentadoras de pesquisa envolvendo seres humanos. 2012.

16. Furlan CS, Guerra FEC, Souza MD de, Oliveira FLS, Fernandes CCDD, Buriola AA. Caracterização de gestantes de alto risco de um ambulatório do interior do oeste paulista. colloquium vitae, v. 10, n. 2, p. 24-34, 2018.

17. Melo AW, Alves JI, Ferreira AA, Souza VS, Maran E. Gestação de alto risco: fatores associados em um município do noroeste paranaense. Rev Saúde Pública Paraná. 2016;17(1):82-91.

18. Medeiros FF, Santos ID de L, Ferrari RAP, Serafim D, Maciel SM, Cardelli AAM. Prenatal follow-up of high-risk pregnancy in the public service. Rev Bras Enferm 2019; 72: 204-11. https:// doi.org/10.1590/0034-7167-2018-0425.

19. Inagaki AD, Cardoso NP, Lopes RJ, Alves JA, Mesquita JR, Araújo KC, Katagiri S. Análise espacial da prevalência de toxoplasmose em gestantes de Aracaju, Sergipe, Brasil. Rev Bras
Ginecol Obstet. 2014;36(12):535-40. https://doi.org/10.1590/ So100720320140005086

20. Avelar MV, Martinez VO, Moura DL de, Barros IA, Primo AA da S, Duarte AO, Soares NM, Lima FW de M. Association between seroprevalence of IgG anti-Toxoplasma gondii and risk factors for infection among pregnant women in Climério de Oliveira Maternity, Salvador, Bahia, Brazil. Revista do Instituto de Medicina Tropical de São Paulo. 2017; 59. https://doi.org/10.1590/S16789946201759090.

21. Silva MG, Vinaud MC, Castro AM. Prevalence of toxoplasmosis in pregnant women and vertical transmission of Toxoplasma gondii in patients from basic units of health from Gurupi, Tocantins, Brazil, from 2012 to 2014. PLoS One 2015; 10(11):1-15.

22. Kamal AM, Ahmed AK, Abdellatif MZM, Tawfik M, Hassan EE. Seropositivity of toxoplasmosis in pregnant women by ELISA at Minia University Hospital, Egypt. Korean J Parasitol 2015; 53(5):605-610.

23. Moura IP da S, Ferreira IP, Pontes AN, Bichara CNC. Conhecimento e comportamento preventivo de gestantes sobre Toxoplasmose no município de Imperatriz, Maranhão, Brasil. Ciênc Saúde Coletiva 2019; 24: 3933-46. https://doi. org/10.1590/1413-812320182410.21702017.

24. Camara, JT, Silva MG, Castro AM. Prevalência de toxoplasmose em gestantes atendidas em dois centros de referência em uma cidade do Nordeste, Brasil. Rev Bras Ginecol Obstet. 2015; 37(2):64-70

25. Mareze M, Benitez A do N, Brandão APD, Pinto-Ferreira F, Miura AC, Martins FDC, Caldart ET, Biondo AW, Freire RL, Mitsuka-Breganó R, Navarro IT. Socioeconomic vulnerability associated to Toxoplasma gondii exposure in southern Brazil. PLoS ONE 2019; 14: e0212375.

26. Rocha EM, Lopes CWG, Ramos RAN, Alves LC. Risk factors for Toxoplasma gondii infection among pregnant women from the State of Tocantins, Northen Brazil. Rev Soc Bras Med Trop 2015; 48(6):773-775.

27. Belluco S, Mancin M, Conficoni D, Simonato G, Pietrobelli M, Ricci A. Investigating the Determinants of Toxoplasma gondii Prevalence in Meat: A Systematic Review and Meta-Regression. Lepczyk CA, editor. Mais Um. San Francisco, CA, EUA: Ciência da Biblioteca Pública; 2016; 11: e0153856.

28. Nunes JT, Gomes KRO, Rodrigues MTP, Mascarenhas MDM. Qualidade da assistência pré-natal no Brasil: revisão de artigos publicados de 2005 a 2015. Cadernos Saúde Coletiva 2016; 24: 252-61. https://doi.org/10.1590/1414-462X201600020171.

29. Tenter AM, Heckeroth AR, Weiss LM. Toxoplasma gondii: from animals to humans. Int J Parasitol . 2000; 30 ( 12-13 ): 1217-1258. 10.1016 / s0020-7519 (00) 00124-7

30. Machacova T, Bartova E, Sedlak K, Slezakova R, Budikova M, Piantedosi D, Veneziano V. Seroprevalence and risk factors of infections with Neospora caninum and Toxoplasma gondii in hunting dogs from Campania region, southern Italy. Parasitol de Folia (Praha). 2016; 6310.14411 / fp.2016.012 